\title{
Low-dimensional antiferromagnetic fluctuations in the heavy-fermion paramagnetic ladder $\mathrm{UTe}_{2}$
}

\author{
W. Knafo, ${ }^{1}$ G. Knebel, ${ }^{2}$ P. Steffens,${ }^{3}$ K. Kaneko, ${ }^{4}$ A. Rosuel, ${ }^{2}$ J.-P. \\ Brison, ${ }^{2}$ J. Flouquet, ${ }^{2}$ D. Aoki,${ }^{5}$ G. Lapertot, ${ }^{2}$ and S. Raymond ${ }^{6}$ \\ ${ }^{1}$ LNCMI-EMFL, CNRS UPR3228, Univ. Grenoble Alpes, Univ. Toulouse, \\ Univ. Toulouse 3, INSA-T, 143 Avenue de Rangueil, 31400 Toulouse, France \\ ${ }^{2}$ Univ. Grenoble Alpes, CEA, Grenoble INP, IRIG, PHELIQS, 38000, Grenoble, France \\ ${ }^{3}$ Institut Laue Langevin, 6 rue Jules Horowitz, BP 156, 38042 Grenoble, France \\ ${ }^{4}$ Materials Sciences Research Center, Japan Atomic Energy Agency, Tokai, Ibaraki 319-1195, Japan \\ ${ }^{5}$ Institute for Materials Research, Tohoku University, Ibaraki 311-1313, Japan \\ ${ }^{6}$ Univ. Grenoble Alpes, CEA, IRIG, MEM, MDN, 38000 Grenoble, France
}

(Dated: June 25, 2021)

\begin{abstract}
Inelastic-neutron-scattering measurements were performed on a single crystal of the heavy-fermion paramagnet $\mathrm{UTe}_{2}$ above its superconducting temperature. We confirm the presence of antiferromagnetic fluctuations with the incommensurate wavevector $\mathbf{k}_{1}=(0,0.57,0)$. A quasielastic signal is found, whose momentum-transfer dependence is compatible with fluctuations of magnetic moments $\mu \| \mathbf{a}$, with a sine-wave modulation of wavevector $\mathbf{k}_{1}$ and in-phase moments on the nearest $\mathrm{U}$ atoms. Low dimensionality of the magnetic fluctuations, consequence of the ladder structure, is indicated by weak correlations along the direction c. These fluctuations saturate below the temperature $T_{1}^{*} \simeq 15 \mathrm{~K}$, in possible relation with anomalies observed in thermodynamic, electrical-transport and nuclear-magnetic-resonance measurements. The absence or weakness of ferromagnetic fluctuations, in our data collected at temperatures down to $2.1 \mathrm{~K}$ and energy transfers from 0.6 to $7.5 \mathrm{meV}$, is emphasized. These results constitute constraints for models of magnetically-mediated superconductivity in $\mathrm{UTe}_{2}$.

PACS numbers: 71.27.+a,74.70.Tx,75.30.Mb,75.40.Gb
\end{abstract}

The discovery of superconductivity at temperatures below $T_{s c} \simeq 1.6 \mathrm{~K}$ in the heavy-fermion paramagnet $\mathrm{UTe}_{2}$, 1, 2] opened a breach in correlated-electron physics. $\mathrm{UTe}_{2}$ was presented as a candidate for topological superconductivity, whose triplet and chiral characters have been proposed [1-11. It turned rapidly out that this system is a unique model to study the electronic correlations and their feedback on magnetism and superconductivity 12 16. A competition between different superconducting pairing mechanisms was indicated from the observation of multiple superconducting phases stabilized near quantum magnetic instabilities under pressure and magnetic field [17-27]. UTe 2 was first suspected to be a nearly-ferromagnet, in which ferromagnetic fluctuations were thought to lead to triplet superconductivity [13. Longitudinal magnetic fluctuations were evidenced by NMR [28] and muon-spin relaxation measurements [29, but these studies could not unambiguously distinguish ferromagnetic and antiferromagnetic fluctuations. The nature of magnetic order induced under pressure [17. also constitutes an open question: ferromagnetism was first suggested [23, 24], but antiferromagnetism was proposed from more recent studies [3, 22, 27, 31. In addition, $\mathrm{UTe}_{2}$ crystallizes in an Immm orthorhombic structure, where the $\mathrm{U}$ atoms form a two-leg ladder structure with legs along $\mathbf{a}$ and rungs along $\mathbf{c}$ [see Fig. 1(a-b)] [15, 26, 32. Therefore, one could suspect that low dimensionality may impact superconducting pairing too.

Inelastic neutron scattering is wavevector-resolved and allows directly determining whether magnetic fluctuations are ferromagnetic or antiferromagnetic. A first neutron-scattering study of $\mathrm{UTe}_{2}$, by time-of-flight spectroscopy on an assembly of 61 crystals offering a total mass of $700 \mathrm{mg}$ and mosaicity of $\simeq 15^{\circ}$, led to the identification of antiferromagnetic fluctuations with the incommensurate wavevectors $\mathbf{k}_{1}=(0,0.57,0)$ and $\mathbf{k}_{2}=(0,0.43,0)$ [expressed in reciprocal lattice units $(\mathrm{rlu})$ and defined within the first Brillouin zone] [4. Here, we present a neutron-scattering study using the tripleaxis spectrometer Thales, at the Institut Laue Langevin in Grenoble. A large single crystal of $\mathrm{UTe}_{2}$ with a mass of $241 \mathrm{mg}$ and a mosaicity of $\lesssim 2^{\circ}$ allowed a fine experimental resolution (see Supplementary Materials [34]). We confirm the presence of magnetic fluctuations with wavevector $\mathbf{k}_{1}$ and do not see clear signatures of ferromagnetic fluctuations with wavevector $\mathbf{k}=0$. A low-dimensional character related with the ladder structure of the $\mathrm{U}$ atoms is emphasized, and the temperature-evolution of the antiferromagnetic fluctuations with wavevector $\mathbf{k}_{1}$ is carefully investigated.

Figure 2 presents energy scans measured at different momentum transfers $\mathbf{Q}$ and $T=2.1 \mathrm{~K}$, for energy transfers $0.6 \leq E \leq 7.5 \mathrm{meV}$. A large signal at the momentum transfer $\mathbf{Q}_{1}=(0,1.43,0)$ indicates the presence of strong antiferromagnetic fluctuations at the incommensurate wavevector $\mathbf{k}_{1}=(0,0.57,0)$ [from $\mathbf{Q}_{1}=\tau-\mathbf{k}_{1}$, where $\tau=(0,2,0)$ is a nuclear Bragg position]. $\mathbf{k}_{1}$ is close to the Brillouin-zone boundary [see Fig. 11(c)]. The 

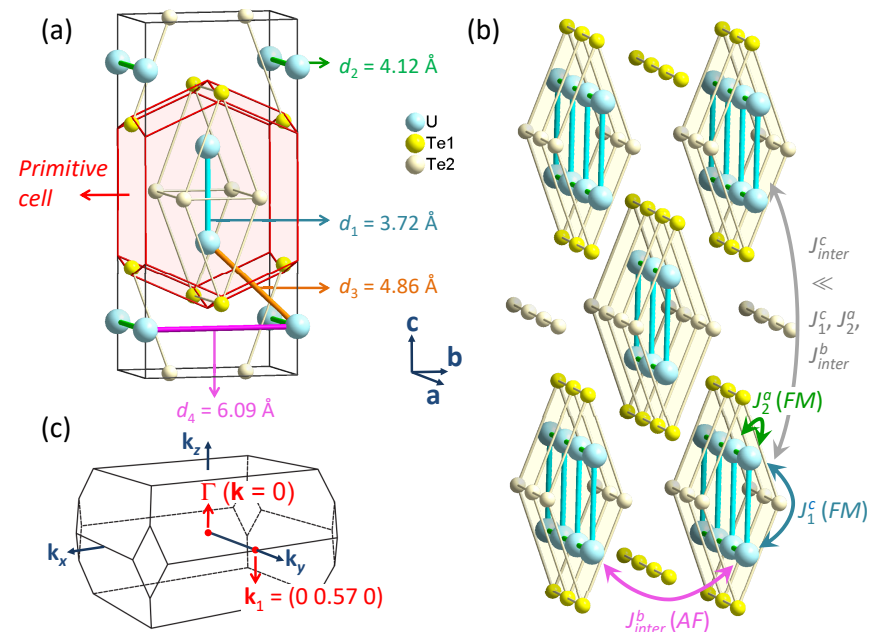

FIG. 1. (a) Orthorhombic and Wigner-Seitz primitive cells, and identification of the four smallest distances (from neutron diffraction at $T=2.7 \mathrm{~K} 32$ ), (b) extended structure emphasizing the ladder structure of $\mathrm{U}$ atoms, and (c) Brillouin zone of $\mathrm{UTe}_{2}$. A phenomenological magnetic-exchange scheme implied in the magnetic-fluctuations mode with wavevector $\mathbf{k}_{1}$ is indicated in (b) (details are given in the text).

momentum transfer $\mathbf{Q}=(0,1.16,1.9)$, chosen far from $\mathbf{Q}_{1}$ (and with the same modulus), is characteristic of a background without magnetic fluctuations. A few points collected at $T=60 \mathrm{~K}$ indicate a nearly-temperature independent background. Spectra at three momentum transfers $\mathbf{Q}=(0,2.1,0),(0,1.07,1.07)$, and $(0,1.07,3.07)$, expected to characterize ferromagnetic fluctuations with wavevector $\mathbf{k} \simeq 0$, present intensities near to the background level 35 . At $\mathbf{Q}=(0,2.1,0)$, the intensity is slightly higher than at the two other ferromagnetic positions, but similar intensities measured at $T=2.1$ and $60 \mathrm{~K}$ indicate a presumably non-magnetic signal. Within the experimental window investigated here $(T \geq 2.1 \mathrm{~K}$ and $0.6 \leq E \leq 7.5 \mathrm{meV}$ ), we do not identify, thus, any clear signature of ferromagnetic fluctuations in $\mathrm{UTe}_{2}$.

Q-dependences of the antiferromagnetic signal centered at $\mathbf{Q}_{1}$, measured at the energy transfer $E=3 \mathrm{meV}$ and at the temperature $T=2.1 \mathrm{~K}$, are presented in Fig. 3. The $\left(0, Q_{k}, 0\right)$ scan shown in Fig. 3(a) indicates an asymmetric anomaly of maximal intensity near $\mathbf{Q}_{1}=$ $(0,1.43,0)$, with a shoulder for $Q_{k}>1.43$. It can be fitted by two Gaussian contributions of full width at half maximum $\kappa=0.24 \mathrm{rlu}$, corresponding to a correlation length $\xi_{b}=3.75 b$, where $b=6.09 \AA$ [32, along the direction b (see Supplementary Materials 34$)$. The contribution centered at the momentum transfer $\mathbf{Q}_{1}=(0,1.43,0)$, corresponding to the wavevector $\mathbf{k}_{1}=(0,0.57,0)$, is twice more intense than that centered at the momentum transfer $\mathbf{Q}_{2}=(0,1.58,0)$, corresponding to the wavevector $\mathbf{k}_{2}=(0,0.42,0)$. In the following, focus will be given to the dominant magnetic-fluctuations mode at wavevector $\mathbf{k}_{1} \cdot\left(0, Q_{k}, 0\right)$ scans at different energy transfers $E$,

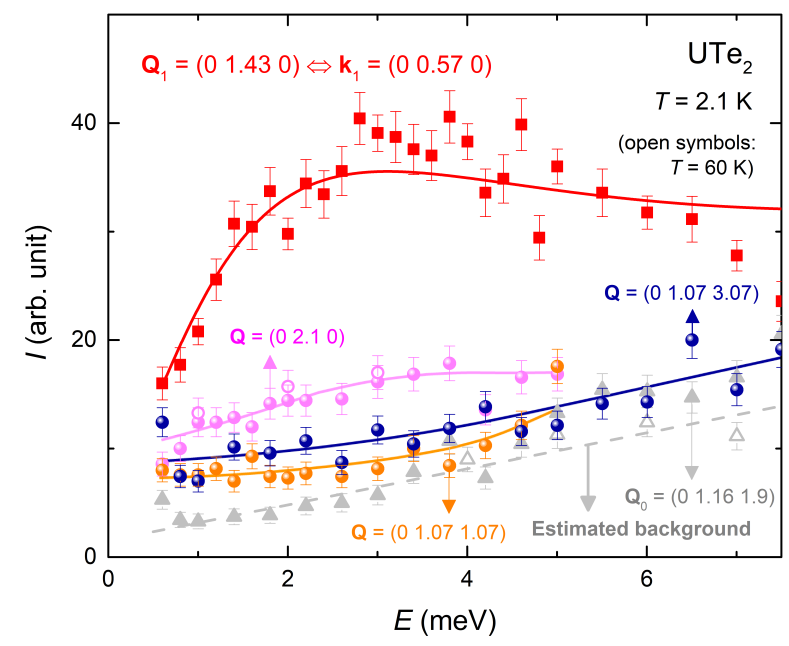

FIG. 2. Energy scans measured at the temperature $T=2.1 \mathrm{~K}$ and the momentum transfers $\mathbf{Q}_{1}=(0,1.43,0)$ characteristic of antiferromagnetic fluctuations (red squares), $\mathbf{Q}=$ $(0,2.1,0), \mathbf{Q}=(0,1.07,1.07)$, and $\mathbf{Q}=(0,1.07,3.07)$ characteristic of ferromagnetic fluctuations (circles), and $\mathbf{Q}=$ $(0,1.16,1.9)$ characteristic of the background (gray triangles). A few points are shown at $T=60 \mathrm{~K}$ and the momentum transfers $\mathbf{Q}=(0,2.1,0)$ and $\mathbf{Q}=(0,1.16,1.9)$ (open symbols). Lines correspond to a fit to the data by a quasielastic Lorentzian shape at the momentum transfer $\mathbf{Q}_{1}$, to a fit to the data by a linear shape at the momentum transfer $\mathbf{Q}=(0,1.16,1.9)$, and to guides to the eyes at the other momentum transfers.

shown in the Supplementary Materials 34, further indicate that the magnetic fluctuations signal at the wavevector $\mathbf{k}_{1}=(0,0.57,0)$ is weakly or not dispersive. In Fig. $3(\mathrm{~b})$, a $\left(0,1.43, Q_{l}\right)$ scan presents a damped sine-wave evolution of the scattered intensity. Best fit to the data is made assuming i) fluctuating magnetic moments $\mu \| \mathbf{a}$ and ii) in-phase magnetic moments on the two $U$ of the primitive cell [see Fig. 11(a) and Supplementary Materials [34]. The sine-wave modulation indicates that, within first approximation, the correlations along $\mathbf{c}$ can be neglected, i.e., that the inter-ladder magnetic coupling along $\mathbf{c}$ is weak. To our knowledge, magnetic correlations along a have not been characterized by neutron scattering so far. However, a first component $k_{1, h}=0$ of the propagation vector $\mathbf{k}_{1}$ is implicitly assumed here and in [4. Indeed, strong correlations along the ladder direction a are expected from the short distance $d_{2}=4.12 \AA$ between $\mathrm{U}$ atoms along $\mathbf{a}$. For these reasons, one can safely expect that the fluctuations with wavevector $\mathbf{k}_{1}$ imply correlations with in-phase fluctuating moments along $\mathbf{a}$. We can further conclude that the antiferromagnetic fluctuations investigated here are low-dimensional, and that they are characterized by a set of equivalent, or nearlyequivalent, lines of wavevectors $\mathbf{k}_{\mathbf{L}}=(0,0.43, \delta)$ and $(0,0.57, \delta)$, which include $\mathbf{k}_{1}$ and $\mathbf{k}_{2}$ (see Supplementary Materials 34). 

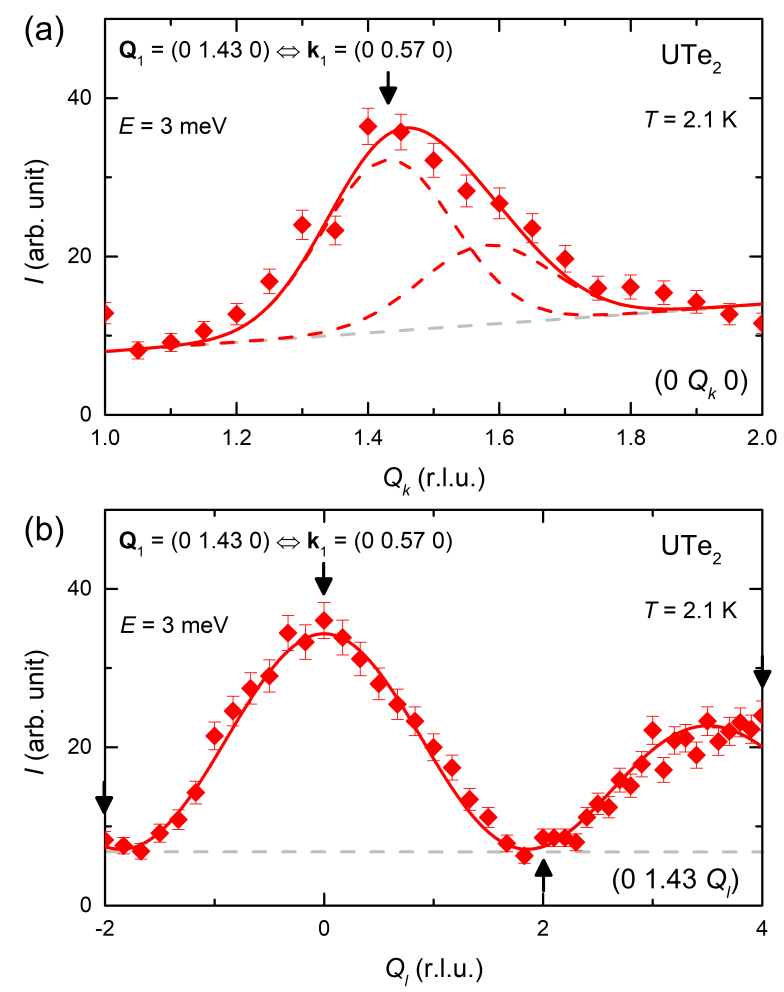

FIG. 3. (a) $\left(0, Q_{k}, 0\right)$ and (b) $\left(0,1.43, Q_{l}\right)$ scans at the energy transfer $E=3 \mathrm{meV}$ and the temperature $T=2.1 \mathrm{~K}$. Gray dashed lines correspond to the estimated background. Red lines correspond to fit to the data as described in [34].

Figure 4(a) shows spectra measured at the momentum transfer $\mathbf{Q}_{1}=(0,1.43,0)$ for a large set of temperatures $T$ from 2.1 to $60 \mathrm{~K}$. The increase of $T$ leads to a progressive decrease and broadening of the antiferromagnetic signal. We fitted data shown in Fig. 4(a) assuming a quasielastic Lorentzian variation of the imaginary part of the dynamical susceptibility $\chi^{\prime \prime}\left(\mathbf{Q}_{1}, E\right)$ (see Supplementary Materials [34). The temperature variations of the relaxation rate $\Gamma\left(\mathbf{k}_{1}\right)$ and of the real part of the static susceptibility $\chi^{\prime}\left(\mathbf{k}_{1}\right)$ extracted from these fits are plotted within log-log scales in Fig. 4(b-c). When the temperature is lowered, the strengthening of antiferromagnetic fluctuations is evidenced by the decrease of $\Gamma\left(\mathbf{k}_{1}\right)$ and the increase of $\chi^{\prime}\left(\mathbf{k}_{1}\right)$, which both saturate at temperatures below $T_{1}^{*} \simeq 15 \mathrm{~K} . T_{1}^{*}$ and the low-temperature value $\Gamma\left(\mathbf{k}_{1}\right) \simeq 2.5 \mathrm{meV} \simeq 2 k_{B} T_{1}^{*}$ are characteristic of the antiferromagnetic fluctuations with wavevector $\mathbf{k}_{1}$.

Anomalies are observed at temperatures near $T_{1}^{*}$ in various physical properties. The bulk magnetic susceptibility $\chi_{a}=M / H$, where $M$ is the magnetization and $\mu_{0} H=0.1 \mathrm{~T}$ a magnetic field applied along the easy magnetic axis a, is compared to $\chi^{\prime}\left(\mathbf{k}_{1}\right)$ in Fig. $4(\mathbf{c})$. Knowing that $\chi_{a}=\chi_{a}^{\prime}(\mathbf{k}=0)$ and $\chi^{\prime}\left(\mathbf{k}_{1}\right)=\chi_{a}^{\prime}\left(\mathbf{k}_{1}\right)$ (since the magnetic fluctuations with wavevector $\mathbf{k}_{1}$ were attributed to magnetic moments $\mu \| \mathbf{a}$ ), both quantities are expected to converge at temperatures at which intersite magnetic correlations have vanished, i.e., presumably $T \gtrsim 100 \mathrm{~K}$. A broad kink in $\chi_{a}$ is observed at the temperature $T_{\chi-a}^{k i n k} \simeq 15 \mathrm{~K} \simeq T_{1}^{*}$. Maxima in the electronic heat capacity [36] and electrical resistivity measured with a current I $\| \mathbf{c}$ [37] and minima in the thermal expansion measured with lengths $\mathbf{L} \| \mathbf{b}, \mathbf{c}$ [36, 38, and thermoelectric power measured with a current I $\mathbf{I}$ a 39] are also observed at a temperature of $\simeq 15 \mathrm{~K}$. These anomalies may result from the development of antiferromagnetic fluctuations with wavevector $\mathbf{k}_{1}$ and their possible feedbacks on the Fermi surface. Figure $4(\mathrm{~d})$ presents a comparison of $\chi^{\prime}\left(\mathbf{k}_{1}\right) / \Gamma\left(\mathbf{k}_{1}\right)$ extracted here and the NMR relaxation rate $1 / T_{1} T$, measured in a magnetic field $\mathbf{H} \| \mathbf{b}$ by Tokunaga et al (for the two sites Te1 and Te2) 28]. These two quantities are dominated by fluctuations of magnetic moments $\mu \| \mathbf{a}$ and vary similarly, increasing with decreasing temperatures before saturating at low temperature. $1 / T_{1} T$ saturates below a temperature of $\simeq 20 \mathrm{~K}$ near $T_{1}^{*}$. Knowing that $1 / T_{1} T$ consists in a sum of $\chi^{\prime}(\mathbf{k}) / \Gamma(\mathbf{k})$ over the reciprocal space (see Supplementary Materials [34]), the magnetic fluctuations at $\mathbf{k}_{1}$ (and its equivalent positions $\mathbf{k}_{L}$ ) may contribute significantly to $1 / T_{1} T$. The slower $T$-variation of $1 / T_{1} T$, in comparison with that of $\chi^{\prime}\left(\mathbf{k}_{1}\right) / \Gamma\left(\mathbf{k}_{1}\right)$, may be due to the contribution of fluctuations at wavevectors far from $\mathbf{k}_{1}$. The question of low-energy ferromagnetic fluctuations (not observed here) and, thus, of their possible contribution to $1 / T_{1} T$, which is sensitive to energies $E \rightarrow 0$, remains open.

Similar relationship between anomalies in bulk properties and magnetic fluctuations were observed in other heavy-fermion paramagnets [40. In the prototypical heavy-fermion paramagnet $\mathrm{CeRu}_{2} \mathrm{Si}_{2}$, the characteristic temperature $T_{1}^{*} \simeq 10 \mathrm{~K}$ of longitudinal antiferromagnetic fluctuations [41] is comparable to those of anomalies in the magnetic susceptibility [42, thermal expansion [43, and NMR relaxation rate [44. The situation in $\mathrm{UTe}_{2}$ is more complex. In addition to the temperature scale of $\simeq 15 \mathrm{~K}$ considered earlier, a second temperature scale of $\simeq 35 \mathrm{~K}$ can be identified from maxima in the magnetic susceptibility $\chi_{b}$ measured with $\mathbf{H} \| \mathbf{b}$ (hard magnetic axis) [45], in the Hall coefficient [39] and electrical resistivity [18] measured with a current $\mathbf{I} \| \mathbf{a}$. Further experiments are needed to determine the relationship between the $15-\mathrm{K}$ and $35-\mathrm{K}$ anomalies, and to test if a second and higher-energy magnetic-fluctuations mode could be present.

The possible coexistence of ferromagnetic and antiferromagnetic couplings in the ladder structure of $\mathrm{UTe}_{2}$ was theoretically discussed in [15], and a first report of antiferromagnetic fluctuations was made in [4]. Thanks to a careful investigation of this antiferromagneticfluctuations mode, we emphasize here its quasielastic and low-dimensional characteristics. It can be visualized as fluctuations of magnetic moments $\mu \| \mathbf{a}$, with the following phenomenological scheme: i) an inter-ladder 

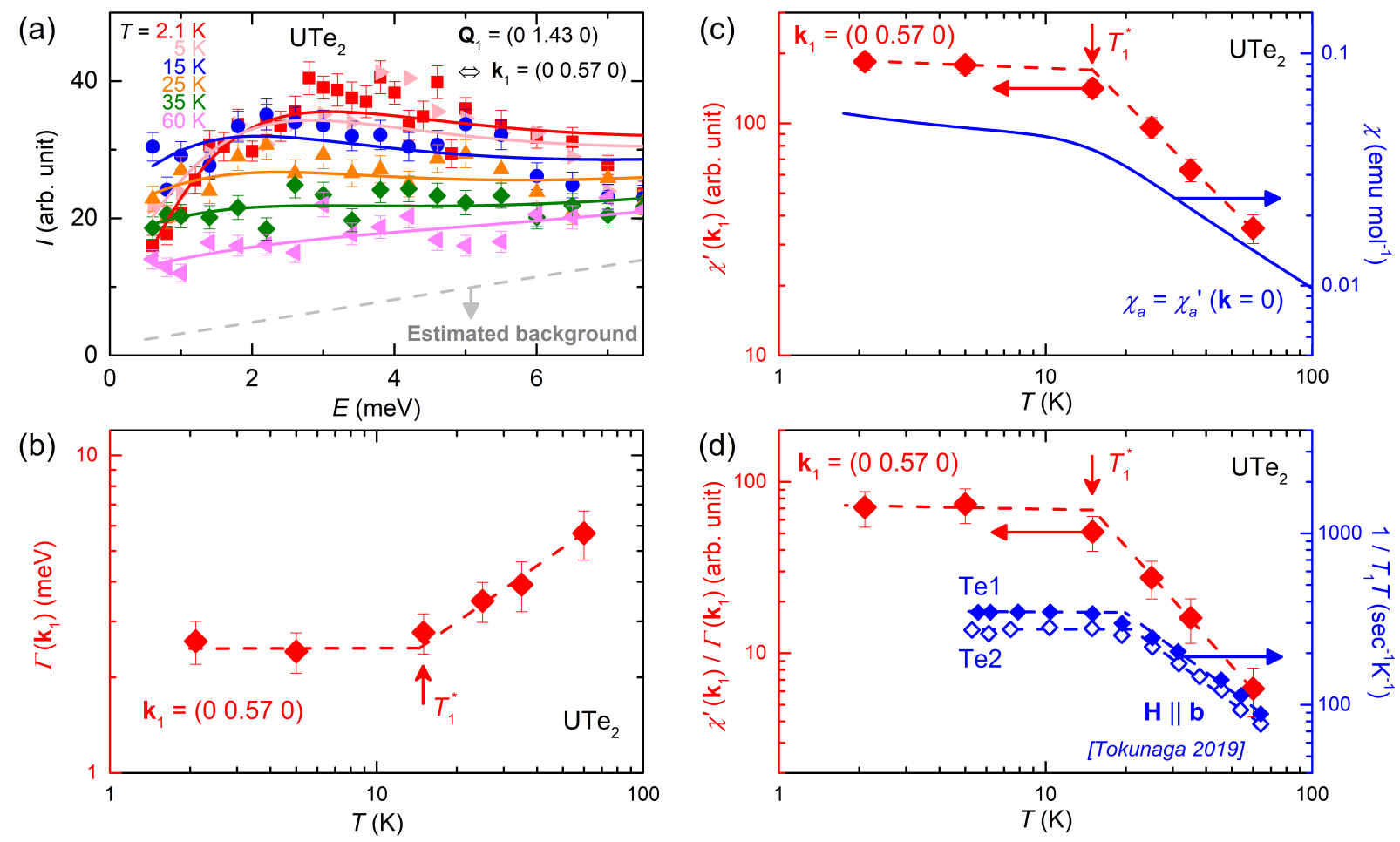

FIG. 4. (a) Energy scans measured at $\mathbf{Q}_{1}=(0,1.43,0)$ and temperatures $T$ from 2.1 to $60 \mathrm{~K}$. Full lines are fits to the data assuming a Lorentzian shape, and the dashed line indicates the estimated background. Temperature dependence, in log-log scales, (b) of the relaxation rate $\Gamma\left(\mathbf{k}_{1}\right)$, (c) of the real part of the static susceptibility $\chi^{\prime}\left(\mathbf{k}_{1}\right)$ and the bulk magnetic susceptibility $\chi_{a}=M / H$ measured in a magnetic field $\mu_{0} \mathbf{H} \| \mathbf{a}$ of $0.1 \mathrm{~T}$, and $(\mathrm{d})$ of the ratio $\chi^{\prime}\left(\mathbf{k}_{1}\right) / \Gamma\left(\mathbf{k}_{1}\right)$ and of the NMR relaxation rate $1 / T_{1} T$ measured on the Te1 and Te2 sites in a magnetic field $\mathbf{H} \| \mathbf{b}$ (from [28]). In panels (c-d), left and right scales have the same number of decades to allow direct comparison between plotted data.

antiferromagnetic coupling $J_{\text {inter }}^{b}(A F)$ ending in sinewave modulation with wavevector $\mathbf{k}_{1}$ of the moments, ii) intra-ladder ferromagnetic couplings $J_{1}^{c}(F M)$ along $\mathbf{c}$ and $J_{2}^{a}(F M)$ along a, and iii) a weak inter-ladder magnetic coupling $J_{\text {inter }}^{c}$ along c [see Fig. 1(a)] [46]. Qmodulations of a magnetic-fluctuations signal similar to those reported here for $\mathrm{UTe}_{2}$ were observed by inelastic neutron scattering in other low-dimensional magnets whose primitive cell contains several magnetic ions, as the magnetic ladder $\mathrm{Sr}_{14} \mathrm{Cu}_{24} \mathrm{O}_{41}$ [47, 48], the layered paramagnet $\mathrm{Sr}_{3} \mathrm{Ru}_{2} \mathrm{O}_{7}$ [49, antiferromagnet $\mathrm{YBa}_{2} \mathrm{Cu}_{3} \mathrm{O}_{6.2}$ [50, and superconductors $\mathrm{YBa}_{2} \mathrm{Cu}_{3} \mathrm{O}_{6.85}$ [51] and $\mathrm{CaKFe}_{4} \mathrm{As}_{4}$ [52. It has been argued that a more robust superconducting pairing is expected for two-dimensional, rather than three-dimensional, itinerant magnets [53. A lowdimensional character of the magnetic fluctuations may, thus, be of importance for the development of superconductivity in $\mathrm{UTe}_{2}$. The question of the role of ferromagnetic fluctuations for the development of a superconducting phase, often suspected to be triplet, needs to be clarified. Ferromagnetic fluctuations were not observed here, within the investigated experimental window $0.6 \leq$ $E \leq 7.5 \mathrm{meV}$, at $T=2.1 \mathrm{~K}$, but Duan et al reported a small signal at the ferromagnetic momentum transfer

\section{ACKNOWLEDGEMENTS}

We acknowledge useful discussions with Y. Tokunaga, K. Ishida, and C. Simon.
$\mathbf{Q}=(0,1,1)$, at $E \simeq 0.4 \mathrm{meV}$ and $T=300 \mathrm{mK}$ [4. An unambiguous experimental evidence for ferromagnetic fluctuations is now needed. We note that in the paramagnet $\mathrm{Sr}_{2} \mathrm{RuO}_{4}$, a former candidate for triplet superconductivity, intense incommensurate antiferromagnetic fluctuations were found to coexist with weak, and broad in Q-space, quasi-ferromagnetic fluctuations [54, 55]. Conversely, triplet superconductivity was proposed to occur in $\mathrm{UPt}_{3}$, where only antiferromagnetic fluctuations were observed [56, 57]. Our finding that the antiferromagnetic fluctuations imply ferromagnetically-coupled U atoms on the ladders may be of importance. Indeed, it is compatible with the proposition of pseudo-triplet superconductivity induced by a ferromagnetic interaction between $\mathrm{U}$ atoms of the ladder rungs, without necessarily implying ferromagnetic fluctuations 8 , 15, 58. In the future, challenges will be to determine precisely the respective roles of antiferromagnetic and ferromagnetic fluctuations and low dimensionality for the stabilization of different superconducting phases in $\mathrm{UTe}_{2}$. The topology and possible low-dimensionality of some Fermi-surface sheets, which set in at low temperatures, also play a role for superconductivity [8, 14, 15] and would merit further consideration too. 
This work was supported by the ANR FRESCO.

[1] S. Ran, C. Eckberg, Q.-P. Ding, Y. Furukawa, T. Metz, S. R. Saha, I.-L. Liu, M. Zic, H. Kim, J. Paglione, and N. P. Butch, Science 365, 684 (2019).

[2] D. Aoki, A. Nakamura, F. Honda, D. Li, Y. Homma, Y. Shimizu, Y. J. Sato, G. Knebel, J.-P. Brison, A. Pourret, D. Braithwaite, G. Lapertot, Q. Niu, M. Vališka, H. Harima, and J. Flouquet, J. Phys. Soc. Jpn. 88, 043702 (2019).

[3] J. Ishizuka, S. Sumita, A. Daido, and Y. Yanase, Phys. Rev. Lett. 123, 217001 (2019).

[4] G. Nakamine, S. Kitagawa, K. Ishida, Y. Tokunaga, H. Sakai, S. Kambe, A. Nakamura, Y. Shimizu, Y. Homma, D. Li, F. Honda, and D. Aoki, J. Phys. Soc. Jpn. 88, 113703 (2019).

[5] T. Metz, S. Bae, S. Ran, I.-L. Liu, Y. S. Eo, W. T. Fuhrman, D. F. Agterberg, S. M. Anlage, N. P. Butch, and J. Paglione, Phys. Rev. B 100, 220504 (2019).

[6] S. Kittaka, Y. Shimizu, T. Sakakibara, A. Nakamura, D. Li, Y. Homma, F. Honda, D. Aoki, and K. Machida, Phys. Rev. Research 2, 032014 (2020).

[7] L. Jiao, S. Howard, S. Ran, Z. Wang, J. O. Rodriguez, M. Sigrist, Z. Wang, N. P. Butch, and V. Madhavan, Nature 579, 523 (2020).

[8] T. Shishidou, H. G. Suh, P. M. R. Brydon, M. Weinert, and D. F. Agterberg, Phys. Rev. B 103, 104504 (2021).

[9] I. M. Hayes, D. S. Wei, T. Metz, J. Zhang, Y. S. Eo, S. Ran, S. R. Saha, J. Collini, N. P. Butch, D. F. Agterberg, A. Kapitulnik, and J. Paglione, arXiv:2002.02539.

[10] S. Bae, H. Kim, Y. S. Eo, S. Ran, I. lin Liu, W. T. Fuhrman, J. Paglione, N. P. Butch, and S. M. Anlage, Nat. Commun. 12, 2644 (2021).

[11] M. Sato and Y. Ando, Rep. Prog. Phys. 80, 076501 (2017).

[12] A. B. Shick, S.-i. Fujimori, and W. E. Pickett, Phys. Rev. B 103, 125136 (2021).

[13] J. Ishizuka and Y. Yanase, Phys. Rev. B 103, 094504 (2021).

[14] L. Miao, S. Liu, Y. Xu, E. C. Kotta, C.-J. Kang, S. Ran, J. Paglione, G. Kotliar, N. P. Butch, J. D. Denlinger, and L. A. Wray, Phys. Rev. Lett. 124, 076401 (2020).

[15] Y. Xu, Y. Sheng, and Y.-f. Yang, Phys. Rev. Lett. 123, 217002 (2019).

[16] K. Machida, Journal of the Physical Society of Japan 89, 033702 (2020).

[17] D. Braithwaite, M. Vališka, G. Knebel, G. Lapertot, J.P. Brison, A. Pourret, M. E. Zhitomirsky, J. Flouquet, F. Honda, and D. Aoki, Commun. Phys. 2, 147 (2019).

[18] W. Knafo, M. Vališka, D. Braithwaite, G. Lapertot, G. Knebel, A. Pourret, J.-P. Brison, J. Flouquet, and D. Aoki, J. Phys. Soc. Jpn. 88, 063705 (2019).

[19] A. Miyake, Y. Shimizu, Y. J. Sato, D. Li, A. Nakamura, Y. Homma, F. Honda, J. Flouquet, M. Tokunaga, and D. Aoki, J. Phys. Soc. Jpn. 88, 063706 (2019).

[20] G. Knebel, W. Knafo, A. Pourret, Q. Niu, M. Vališka, D. Braithwaite, G. Lapertot, M. Nardone, A. Zitouni, S. Mishra, I. Sheikin, G. Seyfarth, J.-P. Brison, D. Aoki, and J. Flouquet, J. Phys. Soc. Jpn. 88, 063707 (2019).

[21] S. Ran, I.-L. Liu, Y. S. Eo, D. J. Campbell, P. Neves,
W. T. Fuhrman, S. R. Saha, C. Eckberg, H. Kim, J. Paglione, D. Graf, J. Singleton, and N. P. Butch, Nat. Phys. 15, 1250 (2019).

[22] D. Aoki, F. Honda, G. Knebel, D. Braithwaite, A. Nakamura, D. Li, Y. Homma, Y. Shimizu, Y. J. Sato, J.-P. Brison, and J. Flouquet, J. Phys. Soc. Jpn. 89, 053705 (2020).

[23] S. Ran, H. Kim, I.-L. Liu, S. R. Saha, I. Hayes, T. Metz, Y. S. Eo, J. Paglione, and N. P. Butch, Phys. Rev. B 101, 140503 (2020).

[24] W. C. Lin, D. J. Campbell, S. Ran, I.-L. Liu, H. Kim, A. H. Nevidomskyy, D. Graf, N. P. Butch, and J. Paglione, npj Quantum Mater. 5, 68 (2020).

[25] G. Knebel, M. Kimata, M. Vališka, F. Honda, D. Li, D. Braithwaite, G. Lapertot, W. Knafo, A. Pourret, Y. J. Sato, Y. Shimizu, T. Kihara, J.-P. Brison, J. Flouquet, and D. Aoki, Journal of the Physical Society of Japan 89, 053707 (2020).

[26] W. Knafo, M. Nardone, M. Vališka, A. Zitouni, G. Lapertot, D. Aoki, G. Knebel, and D. Braithwaite, Commun. Phys. 4, 40 (2021).

[27] D. Aoki, M. Kimata, Y. J. Sato, G. Knebel, F. Honda, A. Nakamura, D. Li, Y. Homma, Y. Shimizu, W. Knafo, D. Braithwaite, M. Vališka, A. Pourret, J.-P. Brison, and J. Flouquet, J. Phys. Soc. Jpn. 90, 074705 (2021).

[28] Y. Tokunaga, H. Sakai, S. Kambe, T. Hattori, N. Higa, G. Nakamine, S. Kitagawa, K. Ishida, A. Nakamura, Y. Shimizu, Y. Homma, D. Li, F. Honda, and D. Aoki, J. Phys. Soc. Jpn. 88, 073701 (2019).

[29] S. Sundar, S. Gheidi, K. Akintola, A. M. Côté, S. R. Dunsiger, S. Ran, N. P. Butch, S. R. Saha, J. Paglione, and J. E. Sonier, Phys. Rev. B 100, 140502 (2019).

[3] S. M. Thomas, F. B. Santos, M. H. Christensen, T. Asaba, F. Ronning, J. D. Thompson, E. D. Bauer, R. M. Fernandes, G. Fabbris, and P. F. S. Rosa, Sci. Adv. 6 (2020).

[31] D. Li, A. Nakamura, F. Honda, Y. J. Sato, Y. Homma, Y. Shimizu, J. Ishizuka, Y. Yanase, G. Knebel, J. Flouquet, and D. Aoki, Journal of the Physical Society of Japan 90, 073703 (2021).

[32] V. Hutanu, H. Deng, S. Ran, W. T. Fuhrman, H. Thoma, and N. P. Butch, Acta Cryst. B 76, 137 (2020).

[4] C. Duan, K. Sasmal, M. B. Maple, A. Podlesnyak, J.-X. Zhu, Q. Si, and P. Dai, Phys. Rev. Lett. 125, 237003 (2020).

[34] See Supplementary Materials for details.

[35] The momentum transfers $\mathbf{Q}=(0,2.1,0),(0,1.07,1.07)$, and $(0,1.07,3.07)$ were chosen near, but not exactly at, the nuclear Bragg positions $\tau=(0,2,0),(0,1,1)$, and $(0,1,3)$ corresponding to magnetic wavevector $\mathbf{k}=0$ to avoid contamination by the nuclear Bragg peaks.

[36] K. Willa et al. to be published.

[37] Y. S. Eo, S. R. Saha, H. Kim, S. Ran, J. A. Horn, H. Hodovanets, J. Collini, W. T. Fuhrman, A. H. Nevidomskyy, N. P. Butch, M. S. Fuhrer, and J. Paglione, arXiv:2101.03102.

[38] S. M. Thomas, C. Stevens, F. B. Santos, S. S. Fender, E. D. Bauer, F. Ronning, J. D. Thompson, A. Huxley, and P. F. S. Rosa, arXiv:2103.09194.

[39] Q. Niu, G. Knebel, D. Braithwaite, D. Aoki, G. Lapertot, G. Seyfarth, J.-P. Brison, J. Flouquet, and A. Pourret, Phys. Rev. Lett. 124, 086601 (2020).

[40] D. Aoki, W. Knafo, and I. Sheikin, C. R. Physique 14, 53 (2013).

[41] W. Knafo, S. Raymond, P. Lejay, and J. Flouquet, Nat. 
Phys. 5, 753 (2009).

[42] P. Haen, J. Flouquet, F. Lapierre, P. Lejay, and G. Remenyi, J. Low Temp. Phys. 67, 391 (1987).

[43] C. Paulsen, A. Lacerda, L. Puech, P. Haen, P. Lejay, J. L. Tholence, J. Flouquet, and A. de Visser, J. Low Temp. Phys. 81, 317 (1990).

[44] K. Ishida, Y. Kawasaki, Y. Kitaoka, K. Asayama, H. Nakamura, and J. Flouquet, Phys. Rev. B 57, R11054 (1998).

[45] S. Ikeda, H. Sakai, D. Aoki, Y. Homma, E. Yamamoto, A. Nakamura, Y. Shiokawa, Y. Haga, and Y. Onuki, J. Phys. Soc. Jpn. 75, 116 (2006).

[46] The phenomenological inter-ladder magnetic couplings $J_{\text {inter }}^{b}$ and $J_{\text {inter }}^{c}$ introduced here may result from two successive 'diagonal' inter-ladder exchanges [along third shortest U-U distance $d_{3}$, see Fig. [1(a-b)].

[47] L. P. Regnault, A. H. Moudden, J. P. Boucher, E. Lorenzo, A. Hiess, A. Vietkin, and A. Revcolevschi, Physica B 259-261, 1038 (1999).

[48] C. Boullier, Ph-D Thesis (University of Grenoble, 2005).

[49] L. Capogna, E. M. Forgan, S. M. Hayden, A. Wildes, J. A. Duffy, A. P. Mackenzie, R. S. Perry, S. Ikeda, Y. Maeno, and S. P. Brown, Phys. Rev. B 67, 012504 (2003).
[50] M. Sato, S. Shamoto, J. M. Tranquada, G. Shirane, and B. Keimer, Phys. Rev. Lett. 61, 1317 (1988).

[51] S. Pailhès, Y. Sidis, P. Bourges, V. Hinkov, A. Ivanov, C. Ulrich, L. P. Regnault, and B. Keimer, Phys. Rev. Lett. 93, 167001 (2004).

[52] T. Xie, Y. Wei, D. Gong, T. Fennell, U. Stuhr, R. Kajimoto, K. Ikeuchi, S. Li, J. Hu, and H. Luo, Phys. Rev. Lett. 120, 267003 (2018).

[53] P. Monthoux and G. G. Lonzarich, Phys. Rev. B 63, 054529 (2001)

[54] A. Pustogow, Y. Luo, A. Chronister, Y.-S. Su, D. A. Sokolov, F. Jerzembeck, A. P. Mackenzie, C. W. Hicks, N. Kikugawa, S. Raghu, E. D. Bauer, and S. E. Brown, Nature 574, 72 (2019).

[55] P. Steffens, Y. Sidis, J. Kulda, Z. Q. Mao, Y. Maeno, I. I. Mazin, and M. Braden, Phys. Rev. Lett. 122, 047004 (2019).

[56] G. Aeppli, E. Bucher, C. Broholm, J. K. Kjems, J. Baumann, and J. Hufnagl, Phys. Rev. Lett. 60, 615 (1988).

[57] H. Tou, Y. Kitaoka, K. Ishida, K. Asayama, N. Kimura, Y. Onuki, E. Yamamoto, Y. Haga, and K. Maezawa, Phys. Rev. Lett. 80, 3129 (1998).

[58] P. W. Anderson, Phys. Rev. B 32, 499 (1985). 


\section{Supplementary Materials: \\ Low-dimensional antiferromagnetic fluctuations in the heavy-fermion paramagnetic ladder $\mathrm{UTe}_{2}$}

In these Supplementary Materials, we present complementary graphs and details concerning the analysis of our data obtained by inelastic neutron scattering on a single crystal of $\mathrm{UTe}_{2}$.

\section{SAMPLE GROWTH AND CHARACTERIZATION}

The 241-mg single-crystalline sample of $\mathrm{UTe}_{2}$ used in this neutron-scattering study was grown using chemical vapor transport method. Figure S1(a) presents a photo of the crystal. Its heat-capacity $C_{p}$, measured at the CEA-Grenoble, is presented as $C_{p} / T$ versus $T$ graph in Fig. S1(b). The onset of superconductivity is visible at the temperature $T_{s c} \simeq 1.6 \mathrm{~K}$. The transition is sharp, with a total width $\Delta T \simeq 0.05 \mathrm{~K}$. This indicates a high quality and small degree of distortion of the crystal. A large increase of $C_{p} / T$ is also observed at temperatures $T<250 \mathrm{mK}$.

$A 3$-scans, where the angle $A 3$ characterizes the rotation of the sample relatively to the vertical axis, around the Bragg positions $\mathbf{Q}=(0,2,0)$ and $\mathbf{Q}=(0,0,4)$ and measured by elastic neutron diffraction at the temperature $T=2 \mathrm{~K}$, are presented in Fig. S2 These scans show that the sample has a mosaicity of $\lesssim 2^{\circ}$.

\section{Q-SCANS AT DIFFERENT ENERGIES}

Complementarily to Fig. 3 of the paper, Fig. S3 presents Q-scans around the antiferromagnetic signal centered at $\mathbf{Q}_{1}=(0,1.43,0)$, measured at energy transfers $E=1,3$, and $5 \mathrm{meV}$ and at the temperature $T=2.1 \mathrm{~K}$. The $\left(0, Q_{k}, 0\right)$ scans shown in Fig. S3(a) present an asymmetric anomaly of maximal intensity near $\mathbf{Q}_{1}=(0,1.43,0)$, with a shoulder for $Q_{k}>1.43$. The $\left(0, Q_{k}, Q_{l}\right)$ scans at constant $Q_{k}$ shown in Fig. S3(b) show a damped sine-wave variation of intensity. These $\mathbf{Q}$ scans performed at different energies indicate that the excitations peaked at $\mathbf{Q}_{1}$ form a rod as a function of energy, and is therefore weakly or not dispersive in the energy range investigated here.

$Q_{k}$ scans were fitted to Gaussian contributions expressed as:

$$
I\left(Q_{k}\right)=I_{0} \exp \left[-4 \ln 2\left(Q_{k} / \kappa\right)^{2}\right]
$$

where $\kappa$ corresponds to the full-width at half maximum (FWHM) of the contribution. A Fourier transformation of Eq. (S1) leads to the real-space distribution of intensity:

$$
I^{\prime}(y)=I_{0}^{\prime} \exp \left[-4 \ln 2\left(y / \xi_{b}\right)^{2}\right]
$$

where $\xi_{b}=4 b \ln 2 /(\pi \kappa)$ is defined as the correlation length in the direction $\mathbf{b}$ of the magnetic fluctuations considered here.
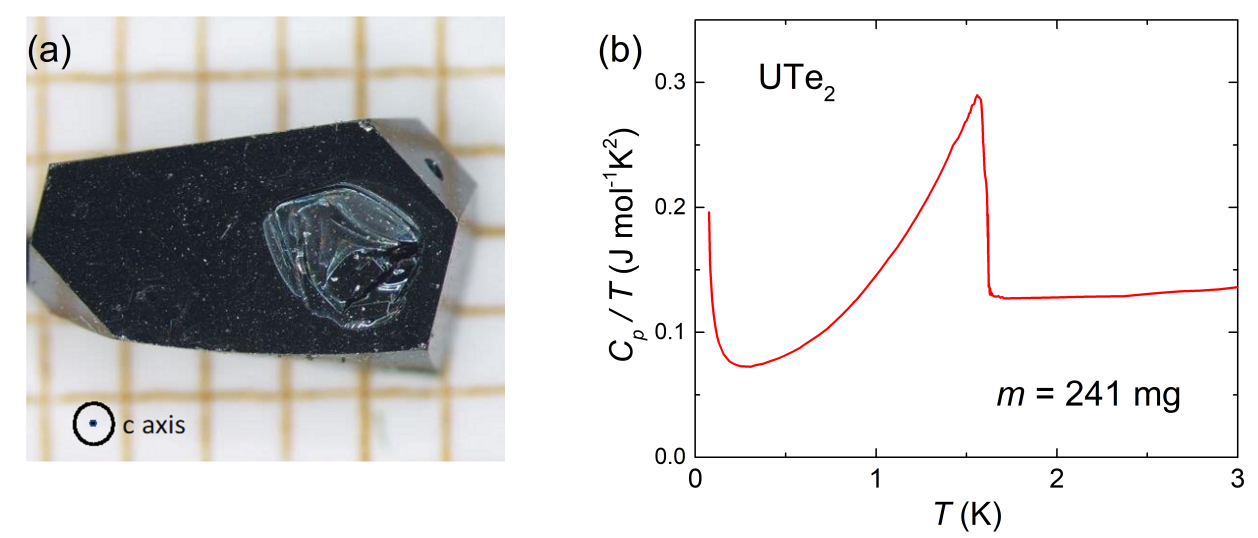

FIG. S1. (a) Photo and (b) heat capacity $C_{p}$, plotted as $C_{p} / T$ versus $T$, of the single crystal used in the present neutron scattering study. 


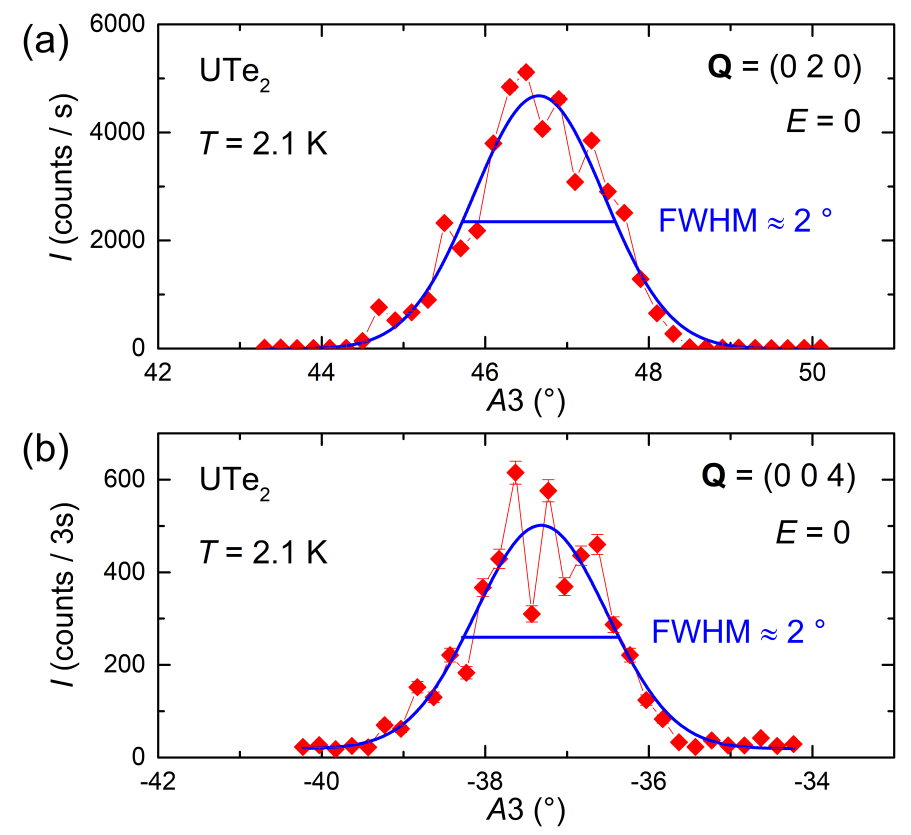

FIG. S2. $A 3$-scans around the Bragg positions $\mathbf{Q}=(0,2,0)$ and $\mathbf{Q}=(0,0,4)$ measured by neutron diffraction at the energy $E=0$ and the temperature $T=2 \mathrm{~K}$.

\section{FIT TO THE $\left(0,1.43, Q_{l}\right)$ SCAN BY A DAMPED SINE-WAVE FUNCTION}

Figure $\mathrm{S} 4$ presents details concerning the fit to the damped sine-wave variation of intensity from the $\left(0,1.43, Q_{l}\right)$ scan made at $T=2.1 \mathrm{~K}$ and shown in Fig. 3(b) of the paper. Data were fitted by a function proportional to the square $F_{m}(\mathbf{Q})^{2}$ of the magnetic structure factor:

$$
F_{m}(\mathbf{Q})^{2}=f_{m}(\mathbf{Q})^{2} \sin ^{2}[\alpha(\mathbf{Q})] \cos ^{2}\left(\pi Q_{l} d_{1} / c\right),
$$

where $f_{m}(\mathbf{Q})$ is the magnetic form factor of individual $\mathrm{U}$ atoms, $\alpha(\mathbf{Q})$ is the angle between $\mathbf{Q}$ and the magnetic moment $\mu$, and the cosine pre-factor is controlled by the magnetic pattern in the primitive cell, with $d_{1}=3.72 \AA$ [see Fig. 1(a) of the paper]. The envelope $f_{m}(\mathbf{Q})^{2} \sin ^{2}[\alpha(\mathbf{Q})]$ and the full fitted function $F_{m}(\mathbf{Q})^{2}$ are plotted assuming the three hypotheses $\mu\|\mathbf{a}, \mu\| \mathbf{b}$, and $\mu \| \mathbf{c}$ in Fig. S4(a), (b) and (c), respectively. While a good fit to the data is obtained assuming $\mu \| \mathbf{a}$, the hypotheses $\mu \| \mathbf{b}$ and $\mu \| \mathbf{c}$ do not permit fitting the data in the whole measured $Q_{l}$ range. In this fit, the magnetic form factor $f_{m}(\mathbf{Q})$ of valence-3 $\mathrm{U}^{3+}$ ions was used [S1, following the conclusions from a photoelectron-spectroscopy study [S2]. We note that a valence 4 corresponding to $\mathrm{U}^{4+}$ ions was also proposed from an x-ray-absorption-spectroscopy experiment and the question of valence in $\mathrm{UTe}_{2}$ remains open $\mathrm{S} 3$. However, the fit done here is almost unaffected by the choice of valence, since the magnetic form factors of $\mathrm{U}^{3+}, \mathrm{U}^{4+}$, but also $\mathrm{U}^{5+}$ ions are very similar $\left[\mathrm{S} 1\right.$. The pre-factor $\cos \left(\pi \mathrm{Q}_{1} \mathrm{~d}_{1} / \mathrm{c}\right)$, which allows a good fit to the data, is expected for in-phase, i.e., ferromagnetically-coupled, magnetic moments on the two $U$ of the primitive cell. We note that the first zero of the pre-factor occurs for $Q_{l}=c /\left(2 d_{1}\right)$, which is accidentally close to $\simeq 2$. Alternative hypotheses can lead to different pre-factors, as $\sin \left(\pi \mathrm{Q}_{1} \mathrm{~d}_{1} / \mathrm{c}\right)$ expected for antiparallel magnetic moments, but do not reproduce the experimental data.

For pedagogical purpose, Fig. S5 shows $Q_{l}$ scans expected for different strengths of the correlations along the direction c, assuming one $U$ atom per primitive cell [Fig. $\mathrm{S5}$ (a)] or assuming in phase magnetic moments on the two $\mathrm{U}$ atoms of the primitive cell of $\mathrm{UTe}_{2}$ [Fig. $[\mathrm{S5}$ (b)]. Well-defined peaks in the case of strong correlations are replaced by broader peaks when the correlations are weakened, ending asymptotically in the envelope given by Eq. (S3) when the correlations vanish.

\section{MAGNETIC FLUCTUATIONS WITH WAVEVECTORS $\mathrm{k}_{1}$ AND $\mathrm{k}_{2}$}

Figure $[$ 6 6 presents series of $\mathbf{Q}$-scans performed at the energy $E=3 \mathrm{meV}$ and the temperature $T=2.1 \mathrm{~K}$. These scans are indicated by arrows in the scattering plane shown in Fig. S5(a). The $\left(0,1.43, Q_{l}\right)$ and $\left(0,1.58, Q_{l}\right)$ scans 

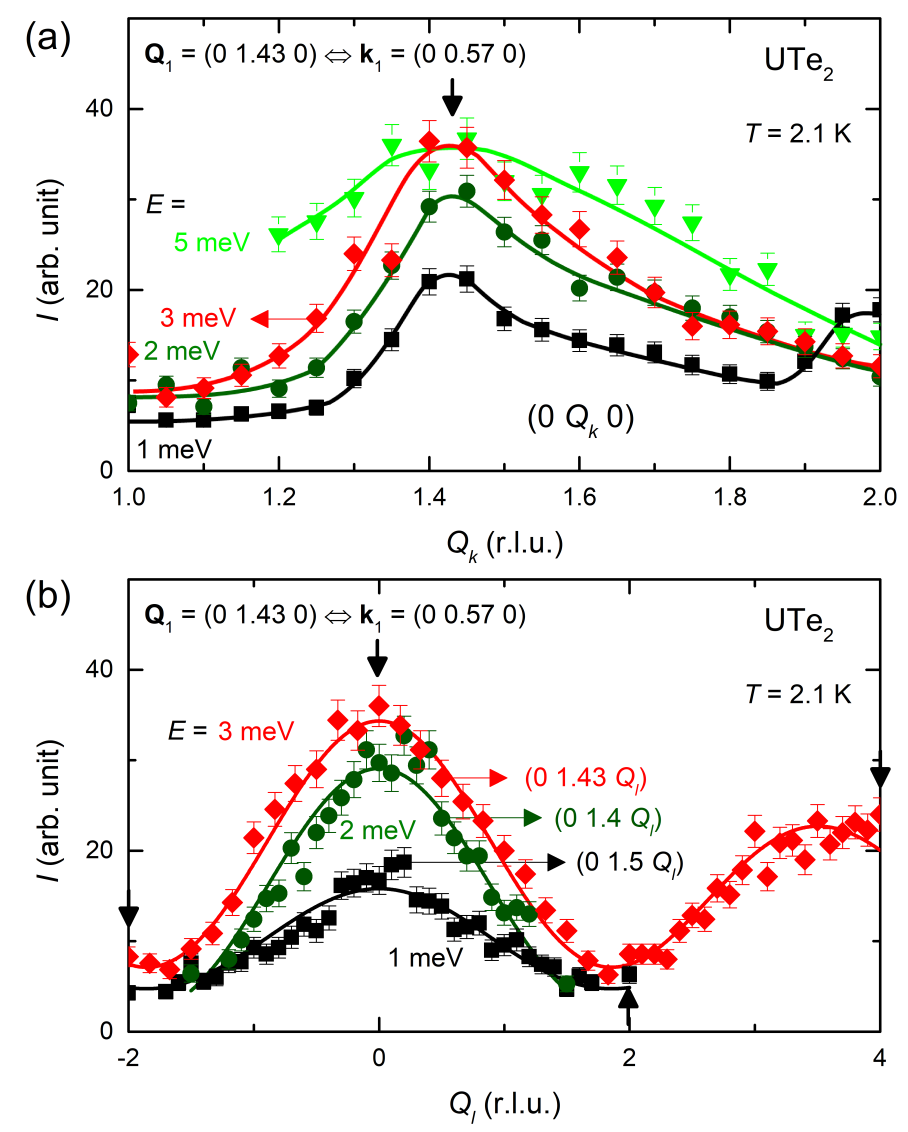

FIG. S3. (a) $\left(0, Q_{k}, 0\right)$ scans at the energy transfers $E=1,3,5 \mathrm{meV}$ and the temperature $T=2.1 \mathrm{~K}$. Full lines are guides to the eyes. (b) $\left(0, Q_{k}, Q_{l}\right)$ scans, where $Q_{k}$ is fixed to a value $1.4 \leq Q_{k} \leq 1.5$, at the energy transfers $E=1,3,5$ meV and the temperature $T=2.1 \mathrm{~K}$. Full lines correspond to fits to the data.

shown in Fig. S6(b) both present a damped sine-wave evolution of the intensity. Each of these scans presents an alternation of $\mathbf{k}_{1}$ and $\simeq \mathbf{k}_{2}$ positions. Within first approximation, their fit to Eq. S3 (see Sect. ) indicates that the antiferromagnetic fluctuations are characterized by a set of wavevectors $\mathbf{k}_{\mathbf{L}}=(0,0.43, \delta)$ and $(0,0.57, \delta)$, which include $\mathbf{k}_{1}$ and $\mathbf{k}_{2}$. These two lines of wavevectors are equivalent for symmetry reasons. However, small deviations are indicated by different scattered intensities at positions corresponding to $\mathbf{k}_{1}$ and $\mathbf{k}_{2}$. These deviations are clear in Fig. S6 (c), which presents $\left(0, Q_{k}, Q_{l}\right)$ scans at constant $Q_{l}=0,1,2,3,4$. Fits made using Gaussian contributions of width $\kappa=0.19-0.24 \mathrm{rlu}$ and centered at positions corresponding to the wavevectors $\mathbf{k}_{1}=(0,0.57,0)$ and $\mathbf{k}_{2}=(0,0.43,0)$ are indicated by full lines in the graphs. These $\left(0, Q_{k}, Q_{l}\right)$ scans (for constant $Q_{l}$ values) show a non-trivial evolution of the two peak intensities:

- the intensity at wavevector $\mathbf{k}_{2}$ probed at the momentum transfer $\mathbf{Q}=(0,1.57,0)$ is smaller than that at the wavevector $\mathbf{k}_{1}$ probed at the momentum transfer $\mathbf{Q}=(0,1.43,0)$ (they should be nearly equal),

- a small intensity remains at wavevector $\mathbf{k}_{2}$ probed at the momentum transfer $\mathbf{Q}=(0,1.43,2)$.

The observed intensities are not compatible with simple phonon contaminations. These deviations may be due to subtle magneto-elastic effects. For instance, the acoustic phonon intensity is expected to be one or two orders of magnitude higher for $Q_{k}=2$ than for $Q_{k}=1$, which may lead to observable coupling with magnetism. A more complete set of experiments, i.e., energy scans at $\mathbf{k}_{2}$ positions and momentum-transfer scans at different energies, possibly at different temperatures, may be needed to study the origin of these effects. Great care to the $\mathbf{Q}$-dependence of the experimental background and of the experimental resolution may be mandatory for such works, for which a larger crystal than the one used here may be needed.

We note that, in their work made on an assembly of 61 single crystals using a time-of-flight spectrometer, Duan et al. observed a vanishing of magnetic fluctuations signal in the line $\left(0, Q_{k}, 2\right)$, in agreement with the cosine term in Eq. (S3) used to fit our data [S4]. 

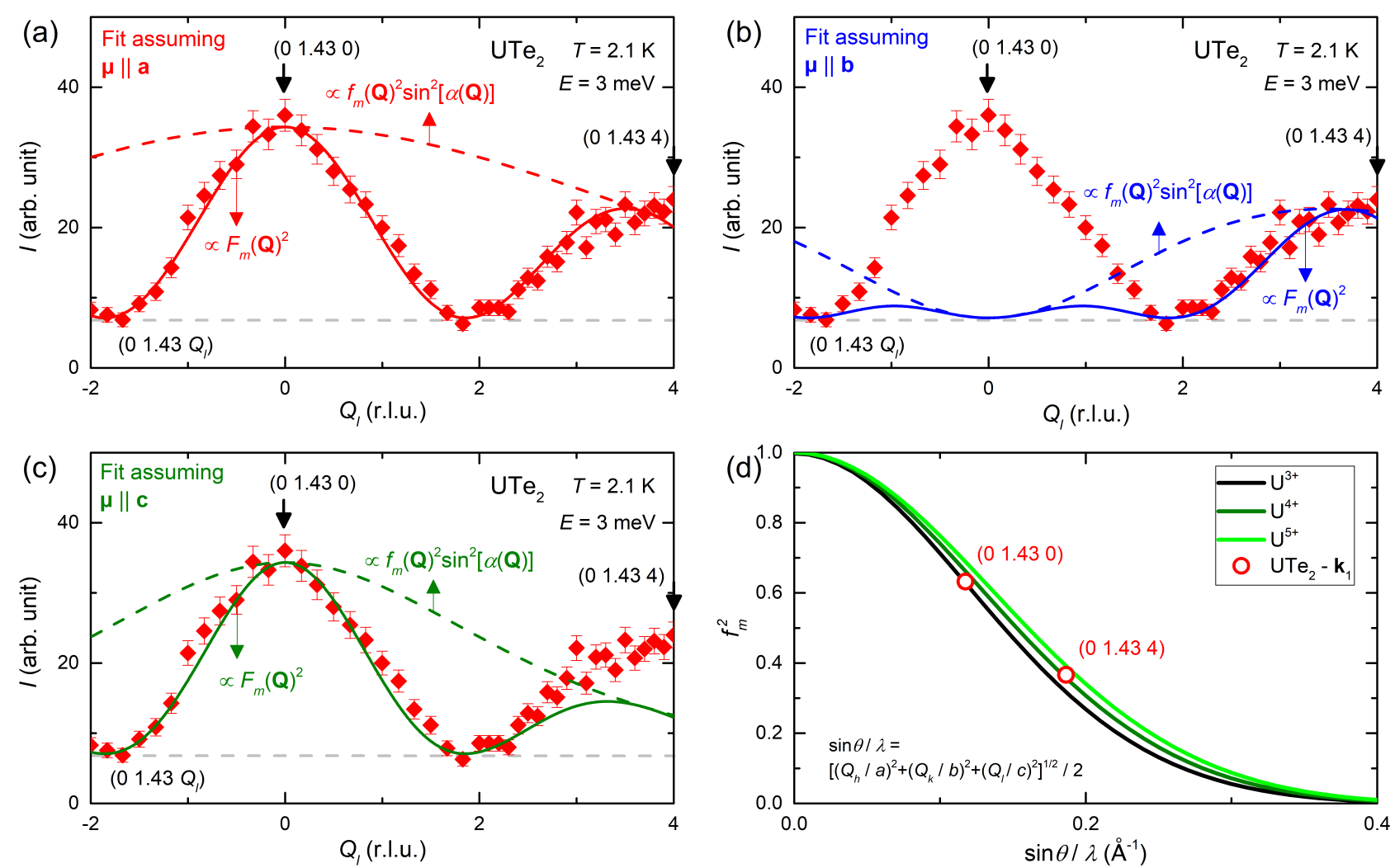

FIG. S4. $\left(0,1.43, Q_{l}\right)$ scan at the energy transfer $E=3 \mathrm{meV}$ and the temperature $T=2.1 \mathrm{~K}$ and its fits assuming magnetic moments (a) $\mu \| \mathbf{a}$, (b) $\mu \| \mathbf{b}$, and (c) $\mu \| \mathbf{c}$. Dashed gray lines correspond to the estimated background, dashed colored lines to the envelope $f_{m}(\mathbf{Q})^{2} \sin ^{2}[\alpha(\mathbf{Q})]$, and full colored lines correspond to fit by $F_{m}(\mathbf{Q})^{2}$ to the data. (d) Variation of magnetic form factor $f_{m}$ with $\sin \theta / \lambda$, where $\theta$ is the scattering angle and $\lambda$ the neutron wavelength, expected for $\mathrm{U}^{3+}, \mathrm{U}^{4+}$, and $\mathrm{U}^{5+}[\mathrm{S} 1$, and comparison with scaled intensities measured on $\mathrm{UTe}_{2}$ at the momentum transfers $(0,1.43,0)$ and $(0,1.43,4)$, for $E=3$ meV and $T=2.1 \mathrm{~K}$.
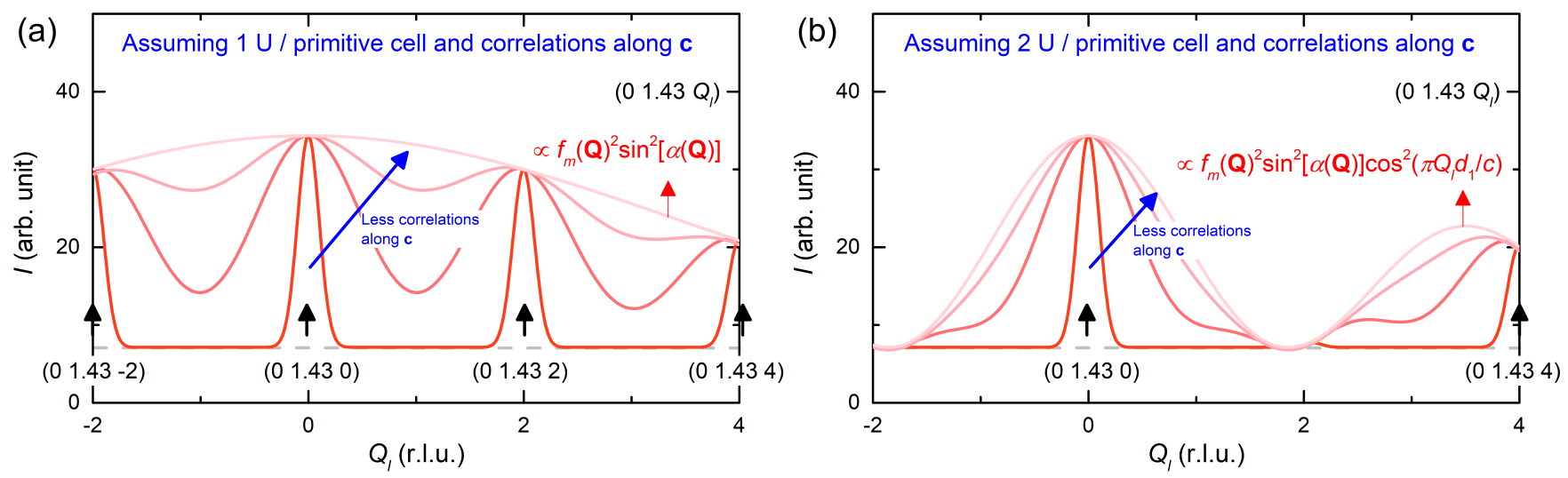

FIG. S5. $Q_{l}$ scans expected for different strengths (from strong to weak) of correlations along the direction c, assuming (a) one $\mathrm{U}$ atom per primitive cell (b) in phase magnetic moments on the two $\mathrm{U}$ atoms of the primitive cell of $\mathrm{UTe}_{2}$.

\section{FIT TO THE ENERGY SCANS BY A QUASIELASTIC LORENTZIAN DYNAMICAL SUSCEPTIBILITY}

The complex magnetic susceptibility, expressed as:

$$
\chi(\mathbf{k}, E)=\chi^{\prime}(\mathbf{k}, E)+i \chi^{\prime \prime}(\mathbf{k}, E),
$$


(a)
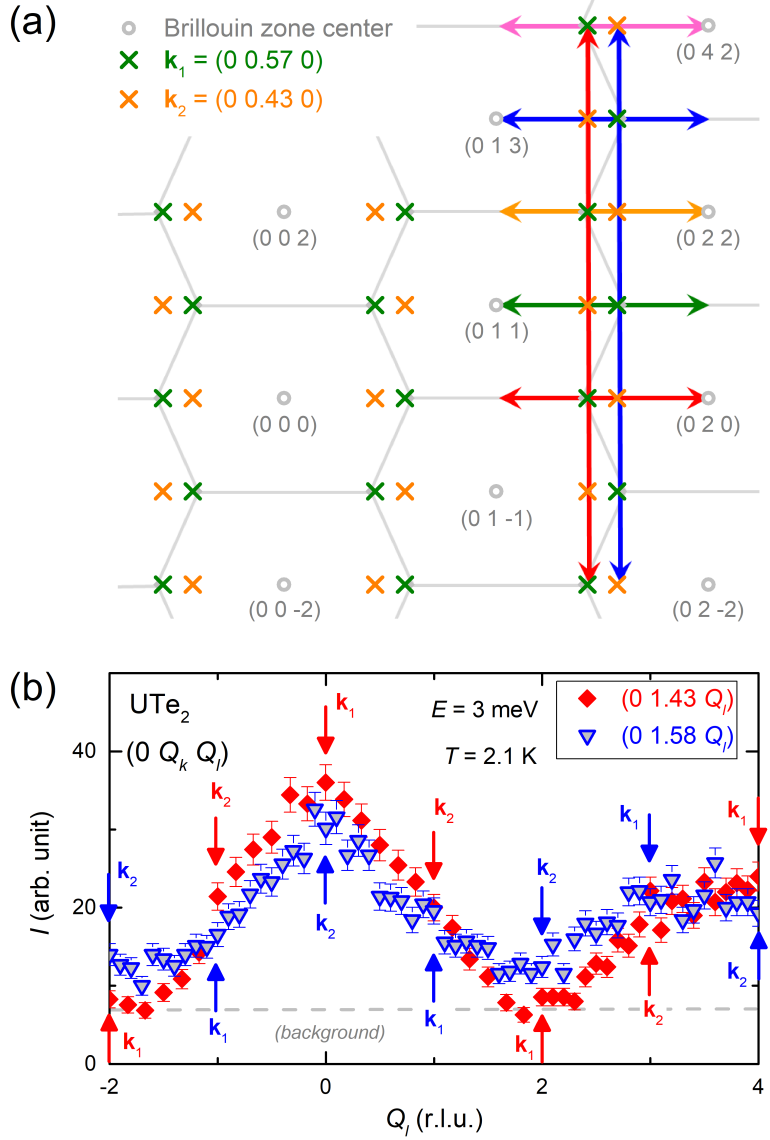

(c)

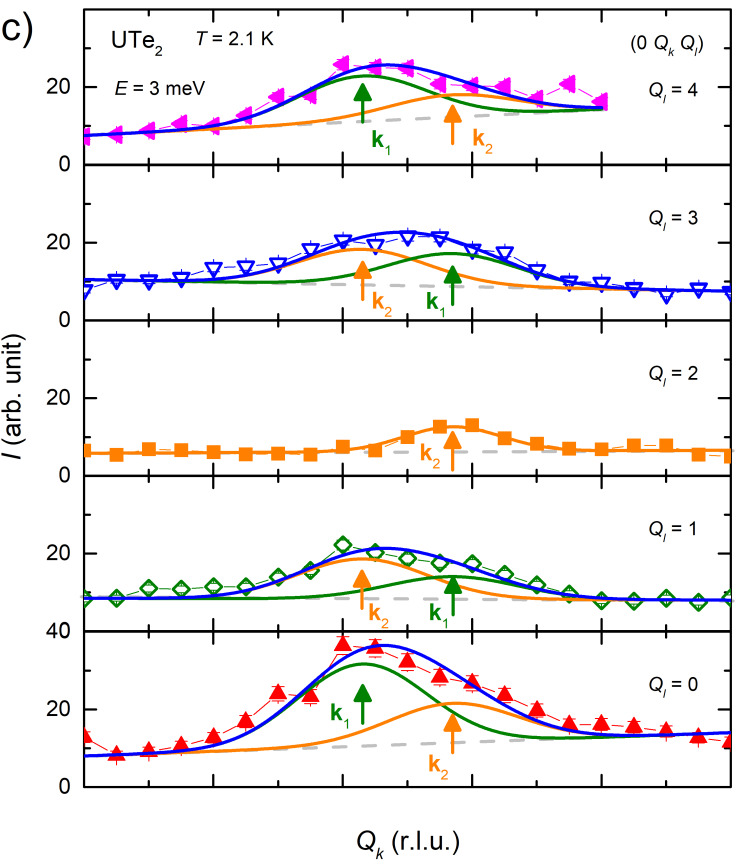

FIG. S6. (a) Scattering plane of the momentum transfers $Q=\left(0, Q_{k}, Q_{l}\right)$ accessed in the neutron experiment. Points at the Brillouin zone centers corresponding to wavevector $\mathbf{k}=0$ are indicated by gray open circles. Points corresponding to the wavevectors $\mathbf{k}_{1}=(0,0.57,0)$ and $\mathbf{k}_{2}=(0,0.42,0)$ are indicated by green and orange crosses, respectively. $\mathbf{Q}$ scans shown in panels (b-c) are indicated by arrows. (b) $\left(0,1.43, Q_{l}\right)$ and $\left(0,1.58, Q_{l}\right)$ scans and (c) $\left(0, Q_{k}, Q_{l}\right)$ scans, with $Q_{l}=0,1,2,3,4$, performed at the energy $E=3 \mathrm{meV}$ and the temperature $T=2.1 \mathrm{~K}$. In panel (c), the lines correspond to fit to the data by one or two Gaussian contributions.

where $\chi^{\prime}(\mathbf{k}, E)$ is its real part and $\chi^{\prime \prime}(\mathbf{k}, E)$ its imaginary part, is generally used to described magnetic fluctuations in the reciprocal space.

Knowing that the neutron intensity $I(\mathbf{Q}, E)$ is related to the imaginary part of the dynamical susceptibility $\chi^{\prime \prime}(\mathbf{Q}, E)$ by:

$$
I(\mathbf{Q}, E)-I_{B G}(E) \propto \frac{F_{m}(\mathbf{Q})^{2}}{1-\exp \left(-E / k_{B} T\right)} \chi^{\prime \prime}(\mathbf{Q}, E),
$$

where $I_{B G}(E)$ is the background, data shown in Fig. 4(a) of the paper were fitted assuming a quasielastic Lorentzian variation of $\chi^{\prime \prime}\left(\mathbf{Q}_{1}, E\right)$ :

$$
\chi^{\prime \prime}\left(\mathbf{Q}_{1}, E\right)=\chi^{\prime}\left(\mathbf{k}_{1}\right) \frac{E / \Gamma\left(\mathbf{k}_{1}\right)}{1+\left(E / \Gamma\left(\mathbf{k}_{1}\right)\right)^{2}},
$$

where $\Gamma\left(\mathbf{k}_{1}\right)$ and $\chi^{\prime}\left(\mathbf{k}_{1}\right)$ are the relaxation rate and the real part of the static susceptibility, respectively, at the wavevector $\mathbf{k}_{1}$ measured at the momentum transfer $\mathbf{Q}_{1}$. We note that a quasielastic Lorentzian variation implies:

$$
\chi(\mathbf{k}, E)=\frac{\chi^{\prime}(\mathbf{k})}{1-i E / \Gamma(\mathbf{k})},
$$

and that the real part of static susceptibility is given by:

$$
\chi^{\prime}(\mathbf{k})=\chi^{\prime}(\mathbf{k}, E=0)=\frac{1}{\pi} \int_{-\infty}^{\infty} \frac{\chi^{\prime \prime}(\mathbf{k})}{E} d E .
$$


For a k-dependent quasielastic-Lorentzian magnetic susceptibility, the NMR spin-lattice relaxation rate $1 / T_{1} T$ is related to $\chi^{\prime}(\mathbf{k})$ and $\Gamma(\mathbf{k})$ by:

$$
\frac{1}{T_{1} T} \propto \sum_{\mathbf{k}}|A(\mathbf{k})|^{2} \frac{\chi^{\prime}(\mathbf{k})}{\Gamma(\mathbf{k})}
$$

where $A(\mathbf{k})$ is a $\mathbf{k}$-dependent hyperfine coupling. A strong $\mathbf{k}$ dependence of the weighting factor $|A(\mathbf{k})|^{2}$ can enhance the contribution at given wavevectors.

[S1] P. J. Brown, Neutron Data Booklet, Chapter 2.5, Magnetic form factors (Institut laue Langevin, Grenoble, Edited by A.J. Dianoux and G. Lander, 2002).

[S2] S.-i. Fujimori, I. Kawasaki, Y. Takeda, H. Yamagami, A. Nakamura, Y. Homma, and D. Aoki, J. Phys. Soc. Jpn. 90, $015002(2021)$.

[S3] S. M. Thomas, F. B. Santos, M. H. Christensen, T. Asaba, F. Ronning, J. D. Thompson, E. D. Bauer, R. M. Fernandes, G. Fabbris, and P. F. S. Rosa, Sci. Adv. 6 (2020).

[S4] C. Duan, K. Sasmal, M. B. Maple, A. Podlesnyak, J.-X. Zhu, Q. Si, and P. Dai, Phys. Rev. Lett. 125, 237003 (2020). 